\title{
The Impact of Virtual Reality in Enhancing the Quality of Life of Pediatric Oncology Patients
}

\author{
Abdullah ALANAZI ${ }^{\mathrm{a}}$, Faisal ASHOUR ${ }^{\mathrm{a}}$, Hanan ALDOSARI ${ }^{\mathrm{a}}$ and Bakheet \\ ALDOSARI $^{\mathrm{a}, 1}$ \\ ${ }^{a}$ College of Public Health and Health Informatics, King Saud bin Abdulaziz University \\ for Health Sciences, Riyadh, Saudi Arabia
}

\begin{abstract}
Pediatric oncology interventions involve many challenges, such as multiple hospitalizations, invasive procedures, and adverse physical and psychological treatment side effects. Treatment burden, including administration of general anesthesia, contributes to high levels of psychological distress among pediatric patients and their families. Virtual reality (VR) is a distraction method, which offers an extremely realistic and interactive virtual environment and helps reduce pain and distress by means of a head-mounted display and headphones. VR is based on two crucial dimensions: immersion and presence, which results in the complete suspension of disbelief that the experience is artificial and allows a greater degree of presence and reaction to the stimulations. The VR technology has become a common practice in scientific and clinical research due to its affordability and ease of use. In pediatric settings, the most widely researched clinical application of VR has focused on the effectiveness of VR distraction therapy in the attenuation of acute pain, anxiety, and distress during invasive medical procedures. It has also been hypothesized to be a nonpharmacological form of analgesia that positively influences the body's intricate pain modulation system during painful medical procedures. In this review, we showed the potential benefits of VR technology during radiotherapy and intrathecal procedures on pediatric oncology patients and its involvement in enhancing their quality of life during and after the treatment. Therefore, a collaboration between researchers, clinicians, and programmers is crucial for the inclusion of VR technology in more clinical procedures, which would consequently enhance the patient's quality of life.
\end{abstract}

Keywords. Virtual Reality, Healthcare, Challenges, Oncology, Pediatrics

\section{Introduction}

Pediatric oncology patients face tremendous challenges during the treatment process since most of the procedures require the administration of general anesthesia (GA), either to reduce anxiety during radiotherapy sessions or for pain management during intrathecal chemotherapy procedures. General anesthesia exerts a tremendous burden on patients as well as the healthcare system. The various side effects of GA include nausea and vomiting, sore throat, dental injury, and confusion. Moreover, it can cause serious complications, such as allergic reactions, respiratory compromise, and death [1].

${ }^{1}$ Corresponding Author, Bakheet Aldosari, College of Public Health \& Health Informatics, King Saud Bin Abdul-Aziz University for Health Sciences, Saudi Arabia; Email: dosarib@gmail.com. 
In addition to the complications, another challenge of delivering radiotherapy to pediatric patients is the recovery process after GA. The recovery process includes three phases: immediate recovery, intermediate recovery, and long-term recovery, which depends on many factors, such as the type of anesthesia, drug factors, and patient characteristics. The recovery should occur within $60-90$ min after GA, which is a considerable amount of time for pediatric patients [2]. Furthermore, there is a need for a larger team comprising anesthesiologists, physicians, nurses, and technicians. Problems are also encountered during anesthesia scheduling owing to the large number of patients and paucity of time. Each patient requires multiple sessions, like five days a week. Therefore, there is a need for targeted, effective interventions that will support pediatric patients in regaining or maintaining their emotional wellbeing and quality of life.

Virtual reality (VR) is an emerging therapeutic tool that engages individuals in a made-up artificial environment by means of a head-mounted display and headphones. The head-mounted display helmet obscures the user's entire real-life visual field and replaces it with a virtual world of visual stimulation, while the headphones replace external auditory sounds with virtual-environment sounds [3]. The factors that contribute to the VR experience are immersion and presence. Immersion considers the action and movement in the virtual space, while presence reflects the involvement of perception in that space. Together, they allow the individual to suspend any disbelief that the experience is artificial [4]. Recently, VR has been used in some areas of clinical practice. The reports from multiple studies conducted in the field of pain management and palliative care are promising [5]. Moreover, VR effectively distracts the patients undergoing wound debridement and reduces pain intensity [6]. VR distraction has also been shown to reduce preoperative anxiety and improve pediatric intra-operative compliance [1]. Although VR was found to significantly reduce pain and distress scores, the effect on anxiety, depression and fear was not significantly attained in pediatric cancer patients [7]. The use of such a technology may decrease the burden of using general anesthesia on patients during medical procedures.

The aim of this review was to determine the potential benefits of VR technology while delivering radiotherapy or intrathecal chemotherapy to pediatric patients. We assessed the delivery time, side effects, and patients' acceptance and satisfaction, which will ultimately enhance their quality of life.

\section{Methodology}

Articles related to VR technology were retrieved from and PubMed databases as well as from Google Scholar. The search strategy included the following MeSH terms ("Virtual Reality"[Mesh]) AND "Quality of Life"[Mesh]), ("Neoplasms"[Mesh] AND "Virtual Reality"[Mesh]). Furthermore, keywords, such as "virtual reality", "healthcare", "oncology", "pediatrics", and "challenges". All articles with these keywords in their title or abstract were screened, with the focus on the limitations and challenges of using VR in medicine. References were hand-searched to identify additional studies not covered by the literature search. Relevant information from each article was extracted, analyzed, and included in the review. 


\section{Results}

Numerous relevant articles on the challenges around the use of VR in medicine were retrieved. In this review, we summarize our findings on VR under two themes: VR as a distraction method and VR in pain management.

\subsection{Virtual Reality as a Distraction method}

Patients typically interacted with different distraction scenarios based on gender and patient age. Distraction methods can be either a game-based software like "Snow World" [2] or an observation-based environment for the user to be relaxed like "Beach or Quit Jungle". The intent of distracting the patient is to have an illusion of going into a different environmental dimension and explore it while they are in their safe zone "next to their parents". The most successful method of using VR relies on the complete suspension of disbelief of being in an artificial/virtual environment so as to achieve a greater degree of presence and reaction to the stimulations. Prior to the initiation of the radiotherapy session, the patients are allowed some time with the VR headsets to adapt to the virtual environment while being in their safe zone environment, which helps in reducing their anxiety level. In general, the critical part is to convince the patients, especially pediatric patients, to wear the goggles before starting the radiotherapy session and then transfer them back to their safe zone without gaining the feeling that they actually went anywhere.

\subsection{Virtual Reality for Pain-related Movement Patterns}

Pediatric patients typically experience increased pain while moving the injured body part, which makes pain management incredibly challenging. The pediatric patients have difficulty accepting treatments like intrathecal chemotherapy procedure [8]. VR has the potential to eliminate that fear by distracting the patient from the pain. Intrathecal chemotherapy is a procedure where the physician will inject a drug into the spinal canal so that it directly reaches the cerebrospinal fluid [8]. Typically, the patients require multiple sessions of drug administration, for example, 3 times a month. Currently, $60 \%$ of the sessions include general anesthesia for patients under 10 years of age, which brings about behavioral changes and is generally not accepted by the patients. The inclusion of the VR technique, considered as an entertainment method, will generate trust and eliminate the fear and pain associated with intrathecal chemotherapy procedure. VR can also be used to modulate behavioral responses during the treatment process. For instance, during the process of locating the spinal canal to inject the drug, the physician will ask the patient to look at the long tree in the artificial environment and to take a deep breath to smell the fresh air. Thus, VR will help the physician in injecting the drug without any difficulties or movement from the patient. In addition, such a procedure will be beneficial for medical institutes in reducing the cost and time associated with the management of a general anesthesia team and for the patients in enhancing their quality of life [10]. 


\section{Discussion and Conclusions}

VR has been applied to different fields, such as dental procedures to increase patient trust, physical therapy to enhance patient recovery, and psychology to enhance the psychological behavior of the patient [11]. However, the medical procedures where VR has proven to be the most effective are radiotherapy and intrathecal procedures. In each case, numerous studies have shown the effectiveness of VR, either as a distraction method or pain management, in enhancing the quality of life of the patient during treatment process. In addition, many studies evaluating the advantages and disadvantages of using VR technologies in pediatric assessments and interventions have demonstrated them to be safe, repeatable, and diversifiable interventions that can be of immense help to healthcare providers. Moreover, VR reduces the anxiety level as well as the experience of aversive stimuli in children. Research on VR interventions for pain and anxiety level holds considerable promise in the medical field. A collaboration between researchers, clinicians, and programmers is crucial for the inclusion of VR technology in more clinical procedures, which would consequently enhance the patient's quality of life.

\section{References}

[1] Hoffman HG, Chambers GT, Meyer WJ, 3rd, Arceneaux LL, Russell WJ, Seibel EJ, Richards TL, Sharar SR, Patterson DR. Virtual reality as an adjunctive non-pharmacologic analgesic for acute burn pain during medical procedures. Ann Behav Med. 2011 Apr;41(2):183-91.

[2] Maani C, Hoffman HG, DeSocio PA, Morrow M, Galin C, Magula J, Maiers A, Gaylord K. Pain control during wound care for combat-related burn injuries using custom articulated arm mounted virtual reality goggles. Journal of CyberTherapy and Rehabilitation. 2008;1:193-198.

[3] Riener R, Harders M. Virtual Reality in Medicine. London: Springer; 2012:1-2.

[4] Cornick JE, Blascovich J. Are virtual environments the new frontier in obesity management? Soc Personal Psychol Compass. 2014;8:650-658.

[5] Kenny MP, Milling LS. The Effectiveness of Virtual Reality Distraction for Reducing Pain: A Metaanalysis. Psychology of Consciousness: Theory, Research and Practice. Advance online publication. 2016.

[6] Keefe FJ, Huling DA, Coggins MJ, Keefe DF, Zachary RM, Herr NR, Hoffman HG. Virtual reality for persistent pain: a new direction for behavioral pain management. Pain, 2012;153(11):2163-2166. https://doi.org/10.1016/j.pain.2012.05.030.

[7] Yap KY, Koh DW, Lee VS, Wong LL. Use of virtual reality in the supportive care management of paediatric patients with cancer. The Lancet Child \& Adolescent Health. 2020 Dec 1;4(12):899-908.

[8] Hoffman HG, Patterson DR, Magula J, Carrougher GJ, Zeltzer K, Dagadakis S, Sharar SR. Waterfriendly virtual reality pain control during wound care. J Clin Psychol. 2004;60:95.

[9] Pullen J, Boyett J, Shuster J, Crist W, Land V, Frankel L, Iyer R, Backstrom L, van Eys J, Harris M. Extended triple intrathecal chemotherapy trial for prevention of CNS relapse in good-risk and poor-risk patients with B-progenitor acute lymphoblastic leukemia: a Pediatric Oncology Group study. J Clin Oncol. 1993 May;11(5):839-49. doi: 10.1200/JCO.1993.11.5.839. PMID: 8487048.

[10] McQuay H, Moore A. An Evidence-Based Resource for Pain Relief. Oxford, UK: Oxford University; 1998.

[11] Pullen J, Boyett J, Shuster J, Crist W, Land V, Frankel L, Iyer R, Backstrom L, van Eys J, Harris M. Journal of Clinical Oncology 1993 11:5, 839-849. 